\title{
Características limnológicas da coluna d'água e dos efluentes de viveiros de criação de camarões-da-amazônia
}

\author{
Gustavo Gonzaga Henry-Silva ${ }^{1}$, Antonio Fernando Monteiro Camargo ${ }^{2}$, Cibele Soares \\ Pontes $^{1}$, Leonardo Kazuya Miyase ${ }^{2}$ \\ ${ }^{1}$ Departamento de Ciências Animais - UFERSA - Mossoró, RN. \\ 2 Departamento de Ecologia - UNESP/CAUNESP - Rio Claro, SP.
}

RESUMO - Os objetivos neste trabalho foram caracterizar os efluentes de viveiros de Macrobrachium amazonicum criados em diferentes densidades de cultivo e analisar a variação nictemeral (24 horas) do perfil vertical da temperatura e da saturação de oxigênio da coluna d'água dos viveiros. O experimento foi realizado durante um período de três meses utilizando-se 12 viveiros retangulares de $100 \mathrm{~m}^{2}$ povoados com camarões-da-amazônia em quatro densidades de cultivo (40, 60, 80 e 100 indivíduos $/ \mathrm{m}^{2}$ ), cada uma avaliada com três repetições. Mensalmente, foi realizado nos viveiros (superfície até o fundo) o acompanhamento da variação nictemeral da temperatura e da saturação de oxigênio dissolvido. Nos efluentes gerados pela criação de camarões foram determinados os valores de N-total, N-amoniacal, N-nitrito, N-nitrato, P-total, P-ortofosfato e turbidez. Foram constatadas estratificações e desestratificações diárias de temperatura e da saturação de oxigênio, independentemente da densidade de cultivo, caracterizando os viveiros como um sistema de circulação polimítico. Quanto maior a densidade de cultivo de camarões-da-amazônia, maiores os valores de P-total, N-total, P-ortofostato, N-amoniacal e turbidez nos efluentes.

Palavras-chave: aquicultura, estratificação, eutrofização, Macrobrachium amazonicum, nictemeral, variação diária

\section{Limnological characteristics of the water column and effluents of Amazon river prawn ponds}

\begin{abstract}
The objective of this research was to characterize the effluents of fish ponds of Macrobrachium amazonicum cultured in different densities of farming and to analyze the daily variation (24 hours) of the vertical temperature and oxygen saturation profile of the water column of the ponds. The study was conducted during a period of three months using 12 rectangular $100 \mathrm{~m}^{2}$ earthen ponds supplied with M. amazonicum individuals in four densities of farming (40, 60, 80 and 100 individuals $/ \mathrm{m}^{2}$ ), each one evaluated with three replicates. Follow-up of the daily variation of the temperature and of the saturation of the dissolved oxygen was monthly carried out in the fish ponds (from the surface to the bottom). It was determined, in the effluent caused by the prawn farming, the values of total-N, ammoniacal-N, nitrite-N, nitrate-N, total-P, orthophosphate-P and turbidity. Daily stratifications and desestratifications of temperature and of the saturation oxygen were recorded, irrespective of farming density, characterizing the ponds as a polimitic system of circulation. The higher the density of cultivation of $M$. amazonicum, the higher the values of total-P, total-N, orthophosphate-P, N-ammoniacal and turbidity.
\end{abstract}

Key Words: aquaculture, daily variation, eutrofication, Macrobrachium amazonicum, nictemeral, stratification

\section{Introdução}

A aquicultura moderna deve se fundamentar no lucro da produção, no desenvolvimento social e na conservação do meio ambiente (NACA/FAO, 2000; Valenti, 2000; Henry-Silva \& Camargo, 2008). Neste contexto, atualmente há uma grande preocupação com a qualidade da água dos viveiros de criação e com os impactos ocasionados pelos efluentes gerados pelas atividades de aquicultura.
As características dos efluentes de aquicultura dependem basicamente da qualidade da água de abastecimento, da quantidade dos alimentos fornecidos, do tempo de residência da água, das espécies criadas e principalmente da densidade de cultivo e da biomassa dos organismos cultivados (Rimon \& Shilo, 1982; Boyd, 2003; Kubitza, 2003). Geralmente, esses efluentes apresentam concentrações elevadas de nitrogênio e fósforo (Kubitza, 2003; Henry-Silva \& Camargo, 2006), que favorecem o aumento 
da comunidade fitoplanctônica, alterando a dinâmica da temperatura e do oxigênio dissolvido na coluna d'água dos viveiros (Midlen \& Redding, 1998; Mires, 1995). Neste contexto, os viveiros de criação tendem a ser ambientes eutrofizados, onde podem ocorrer estratificações térmica e química da coluna d'água. Durante o período de estratificação, o hipolímnio pode apresentar baixas concentrações de oxigênio dissolvido, comprometendo o desenvolvimento e a produtividade dos organismos bentônicos, especialmente de camarões.

Atualmente, os crustáceos vêm se destacando entre os organismos cultivados, de modo que a criação de camarões-d'água-doce tornou-se um dos setores que mais crescem no mundo (Valenti, 2000). No Brasil, existem espécies nativas com potencial para a criação em cativeiro, como o Macrobrachium amazonicum (Valenti \& MoraesRiodades, 2004; New, 2005). No entanto, o sucesso da produção depende do conhecimento biológico da espécie, da avaliação dos impactos gerados pela atividade e da manutenção da qualidade de água dos viveiros (Boyd \& Zimmermann, 2000; Moraes-Valenti \& Valente, 2007). Neste contexto, os objetivos neste trabalho foram caracterizar os efluentes provenientes de viveiros de M. amazonicum criados em diferentes densidades de cultivo e analisar a variação nictemeral (24 horas) do perfil vertical da temperatura e da saturação de oxigênio da coluna d'água dos viveiros.

\section{Material e Métodos}

O experimento foi realizado no Setor de Carcinicultura do Centro de Aquicultura da Universidade Estadual Paulista $\left(21^{\circ} 15^{\prime} 22^{\prime \prime} \mathrm{S}\right.$ e $\left.48^{\circ} 18^{\prime} 48^{\prime \prime} \mathrm{W}\right)$ e as variáveis limnológicas foram obtidas durante um período de três meses, entre janeiro e março de 2006. O delineamento experimental foi constituído de quatro densidades de cultivo $\left(40,60,80\right.$ e 100 indivíduos $\left./ \mathrm{m}^{2}\right)$, cada uma avaliada com três repetições. Foram utilizados 12 viveiros de $100 \mathrm{~m}^{2}$, escavados com fundo de terra, sem revestimento nas laterais e profundidade de 1,2 m. As pós-larvas de M. amazonicum foram provenientes do estoque de reprodutores do Setor de Carcinicultura do CAUNESP. A biomassa média inicial dos indivíduos em todas as densidades de cultivo foi de $0,02 \mathrm{~g}$. As biomassas iniciais e finais dos camarões foram, respectivamente, $80 \mathrm{~g}$ e $12,3 \mathrm{~kg}$ na densidade de $40 ; 120 \mathrm{~g}$ e $13,8 \mathrm{~kg}$ na densidade de $60 ; 160 \mathrm{ge} 14,8 \mathrm{~kg}$ na densidade de 80 ; e $200 \mathrm{~g} \mathrm{e} 21,2 \mathrm{~kg}$ na densidade de 100 indivíduos $/ \mathrm{m}^{2}$ ). O arraçoamento foi realizado duas vezes ao dia entre $7 \mathrm{~h} 30 \mathrm{e}$ $8 \mathrm{~h} 30$ e $16 \mathrm{~h}$ e $17 \mathrm{~h}$ em iguais porções.
O povoamento dos viveiros com juvenis de aproximadamente 18 dias após a metamorfose em póslarvas foi realizado em 12 de dezembro de 2005. Até o dia 13 de janeiro, a taxa diária de arraçoamento de cada viveiro foi de $2,5 \mathrm{~g} / \mathrm{m}^{2}$. A partir daí, foi fornecida $9 \%$ da biomassa dos camarões, reduzindo a taxa para $7 \%$ da biomassa a partir do dia 14 de fevereiro e para 5\% a partir de 14 de março. A alimentação foi feita com ração peletizada para camarões marinhos Fri-Ribe HD 35 de alta densidade (Tabela 1). Durante todo o experimento, não foram utilizados aeradores nas unidades experimentais.

Mensalmente, todo dia 28 de cada mês, foram obtidas dentro dos viveiros as medidas de temperatura e saturação de oxigênio com um medidor portátil. As medidas foram realizadas na superfície $(0,0 \mathrm{~m})$ e aos 0,$15 ; 0,30 ; 0,45 ; 0,60$; 0,75; 0,90 e 1,05 m (fundo), em intervalos de 3 horas a partir das $9 \mathrm{~h}$ até que se completassem 24 horas de coleta. No início do experimento, foi sorteado um viveiro de cada de cada uma das densidades de cultivo para acompanhamento dos perfis verticais de temperatura e saturação de oxigênio.

Também mensalmente, entre $9 \mathrm{~h} \mathrm{e} 10 \mathrm{~h}$, foram coletadas amostras dos efluentes de cada viveiro de engorda de camarões $(n=3)$ e da água de abastecimento. Nessas amostras, foram determinadas as concentrações de N-amoniacal, conforme descrição de Koroleff (1976); e os valores de $\mathrm{N}$-total, N-nitrito, N-nitrato, de acordo com Mackereth et al. (1978). As concentrações de P-total e P-ortofosfato foram obtidas segundo o método proposto por Golterman et al. (1978) e os valores de turbidez com auxílio de um multissensor da marca Horiba, modelo U-10. Com os valores médios (variação temporal) dessas variáveis, foi realizada uma Análise de Componentes Principais - ACP (Ludwig \& Reynolds; 1988), após a normalização dos dados com a aplicação da raiz quadrada.

Tabela 1 - Composição da ração utilizada no experimento ${ }^{1}$

\begin{tabular}{lc}
\hline & Nível de garantia \\
\hline Umidade (máxima) & $13 \%$ \\
Proteina bruta (mínimo) & $35 \%$ \\
Extrato etéreo (mínimo) & $6 \%$ \\
Fibra bruta (máximo) & $6 \%$ \\
Matéria mineral (máximo) & $13 \%$ \\
Cálcio (máximo) & $3 \%$ \\
Fósforo (mínimo) & $0,7 \%$ \\
\hline
\end{tabular}

${ }^{1}$ Ração comercial Fri-Ribe HD 35 (alta densidade). 


\section{Resultados e Discussão}

Em todas as densidades de cultivo, a temperatura e a saturação de oxigênio aumentaram no período da tarde (15 e 18 h). No início do período da manhã (6 h), os valores de oxigênio dissolvido foram menores e corresponderam ao efeito dos processos de respiração dos organismos aquáticos e da decomposição da matéria orgânica ocorridos durante a noite. $\mathrm{O}$ maior resfriamento da coluna d'água também ocorreu às $6 \mathrm{~h}$ e, com a incidência de raios solares no período da manhã, iniciaram-se o aquecimento da coluna d'água e o aumento da fotossíntese pelo fitoplâncton, que acarretou no consumo de $\mathrm{CO}_{2}$ e na produção de oxigênio. Portanto, a quantidade de oxigênio medida no período da tarde representou o saldo entre o que foi produzido pelo fitoplâncton e o que foi gasto nos processos de respiração de decomposição.

Durante o período da tarde, os valores de temperatura e de saturação de oxigênio no fundo dos viveiros foram menores se comparados aos observados na superfície. No fundo, a menor incidência de luz provavelmente limitou a fotossíntese e a redução da produção de oxigênio. Além disso, o consumo deste gás nesta região tende a ser maior devido à respiração dos camarões, que possuem hábito bentônico, e devido à decomposição dos restos de ração e fezes produzidas por esses organismos. Provavelmente, a menor incidência solar também contribuiu para a menor temperatura da água no fundo dos viveiros.

De acordo com Payne (1986), diferenças de apenas $0,5^{\circ} \mathrm{C}$ entre a superfície e a parte inferior da coluna d'água podem permitir que um ambiente aquático tropical apresente estratificação térmica relativamente estável, pois as temperaturas elevadas fazem com que pequenas variações na temperatura da água correspondam a grandes diferenças em sua densidade. Dessa forma, houve um processo de estratificação e desestratificação térmica diária em todos os viveiros, independentemente da densidade de cultivo dos camarões; a maior amplitude de variação da temperatura entre superfície e fundo ocorreu no mês de fevereiro no período da tarde ( $15 \mathrm{~h}$ ) nos viveiros com maiores densidades de cultivo ( 80 e 100 indivíduos $/ \mathrm{m}^{2}$ ). $\mathrm{Na}$ densidade 80 , os valores de temperatura na superfície foram de 33 e $27,7^{\circ} \mathrm{C}$ no fundo $(1,05 \mathrm{~m})$, enquanto na densidade de 100 indivíduos $/ \mathrm{m}^{2}$, os valores foram de $33,5^{\circ} \mathrm{C}$ na superfície e de $27,8^{\circ} \mathrm{C}$ no fundo. Os resultados evidenciam ainda que o processo de estratificação térmica da coluna d'água dos viveiros inicia-se por volta das $12 \mathrm{~h}$ e atinge seu valor máximo às $15 \mathrm{~h}$, mantendo-se até por volta das $18 \mathrm{~h}$. Os valores de temperatura obtidos às $21 \mathrm{~h}$, no entanto, comprovam a quebra da estratificação térmica e isotermia da coluna d'água em todas as densidades de cultivo e essa homogeneidade da temperatura na coluna d'água permaneceu até por volta das $6 \mathrm{~h}$ da manhã (Figuras 1 a 3). Rimon \& Shilo (1982) também encontraram variação de até $5^{\circ} \mathrm{C}$ entre a superfície e o fundo de viveiros de criação de peixes em Israel. Esteves et al. (1988), estudando uma lagoa costeira tropical com profundidade média de 1 metro (Lagoa Iodada, Rio de Janeiro) identificaram estratificação térmica acentuada da coluna d'água durante o período iluminado do dia e desestratificação no período noturno.

Os maiores valores de saturação de oxigênio foram observados nas densidades de 80 e 100 indivíduos $/ \mathrm{m}^{2}$. Nessas densidades de cultivo, a amplitude de variação desse parâmetro durante o período de 24 horas na superfície dos viveiros foi maior que nas densidades 40 e 60 (Figuras 4 a 6). Provavelmente as maiores quantidades de camarões e o maior arraçoamento nas densidades de cultivo mais elevadas contribuíram para o aumento do fitoplâncton, que, por meio da fotossíntese, proporcionou acréscimo nas concentrações de oxigênio durante o dia.

De modo geral, as maiores amplitudes de variação da saturação de oxigênio entre superfície e fundo dos viveiros ocorreram às $18 \mathrm{~h}$ nos três meses estudados. Nas menores densidades de cultivo, as maiores amplitudes de variação foram encontradas em março, ou seja, $157 \%$ na superfície e $62 \%$ no fundo na densidade 40 indivíduos $/ \mathrm{m}^{2}$ e $155 \%$ na superfície e $61 \%$ no fundo na densidade 60 indivíduos $/ \mathrm{m}^{2}$. $\mathrm{Na}$ densidade de 80 indivíduos $/ \mathrm{m}^{2}$, a maior diferença foi constatada em fevereiro às $18 \mathrm{~h}(160 \%$ a $30 \mathrm{~cm}$ e $116 \%$ no fundo). No viveiro com maior densidade de cultivo (100 indivíduos $/ \mathrm{m}^{2}$ ), a maior diferença entre superfície e fundo da coluna d'água também foi observada em fevereiro, quando os valores foram de $185 \%$ a $30 \mathrm{~cm} \mathrm{de}$ profundidade e $114 \%$ no fundo. Esses resultados evidenciam que os viveiros com maiores densidades de cultivo (80 e 100 indivíduos $/ \mathrm{m}^{2}$ ) eutrofizaram mais rapidamente que os viveiros com densidades 40 e 60 indivíduos $/ \mathrm{m}^{2}$, provavelmente em decorrência da maior quantidade e biomassa de camarões e do maior aporte de ração fornecida.

Os valores de saturação de oxigênio durante as 24 horas apresentaram gradiente vertical, independentemente da densidade de cultivo, ou seja, valores mais altos durante o dia e menores durante a noite em todas as profundidades. Esse perfil do tipo clinogrado deve-se à alta taxa fotossintética na zona eufótica durante o dia e pelo consumo 
Temperatura $\left({ }^{\circ} \mathrm{C}\right)$
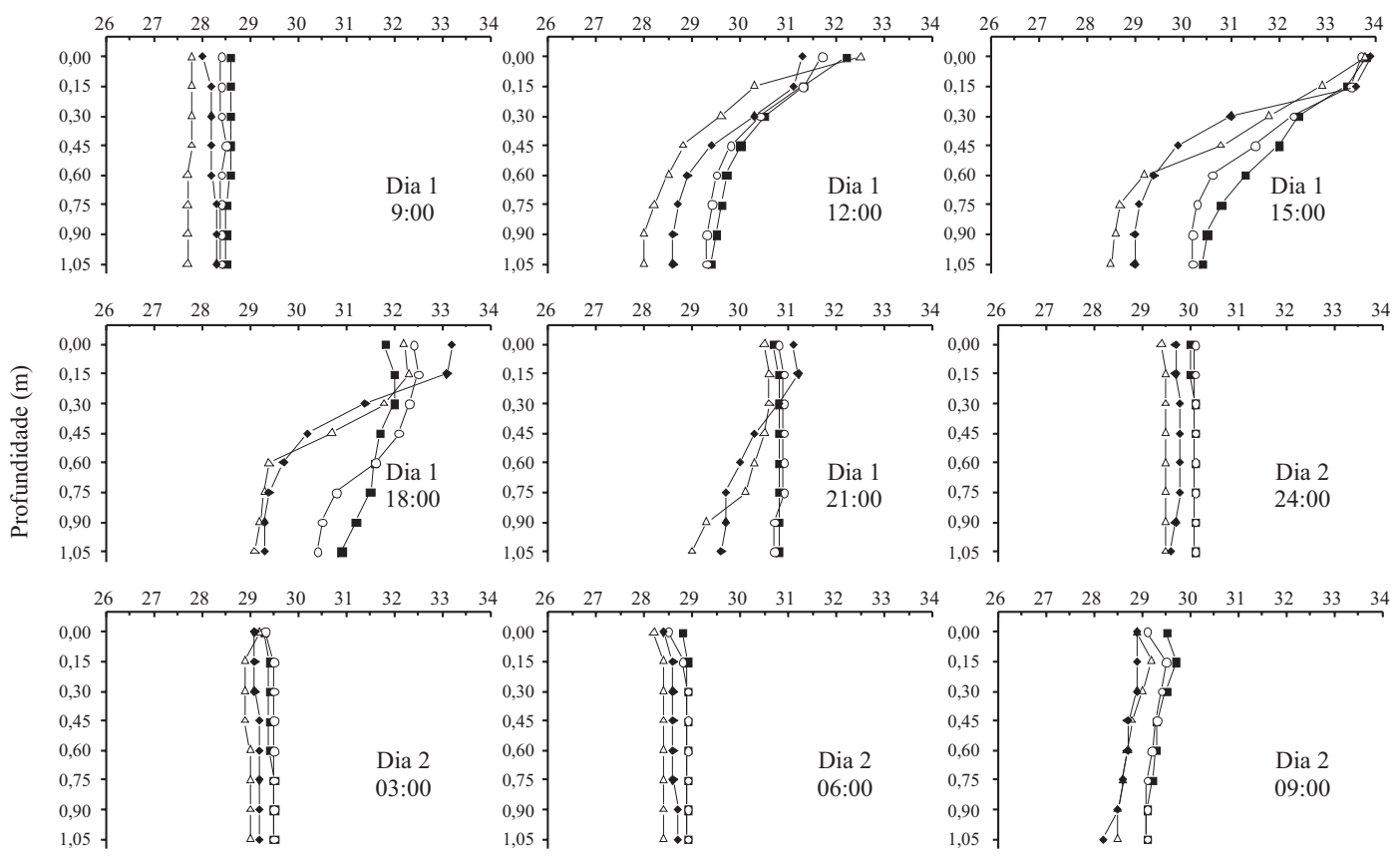

= 40 indivíduos $/ \mathrm{m}^{2} ; \bigcirc=60$ indivíduos $/ \mathrm{m}^{2} ; \Delta=80$ indivíduos $/ \mathrm{m}^{2} ; \bullet=100$ indivíduos $/ \mathrm{m}^{2}$

Figura 1 - Variação diária (padrão nictemeral) dos perfis verticais de temperatura de viveiros de camarões-da-amazônia no mês de janeiro de 2008.

Temperatura $\left({ }^{\circ} \mathrm{C}\right)$
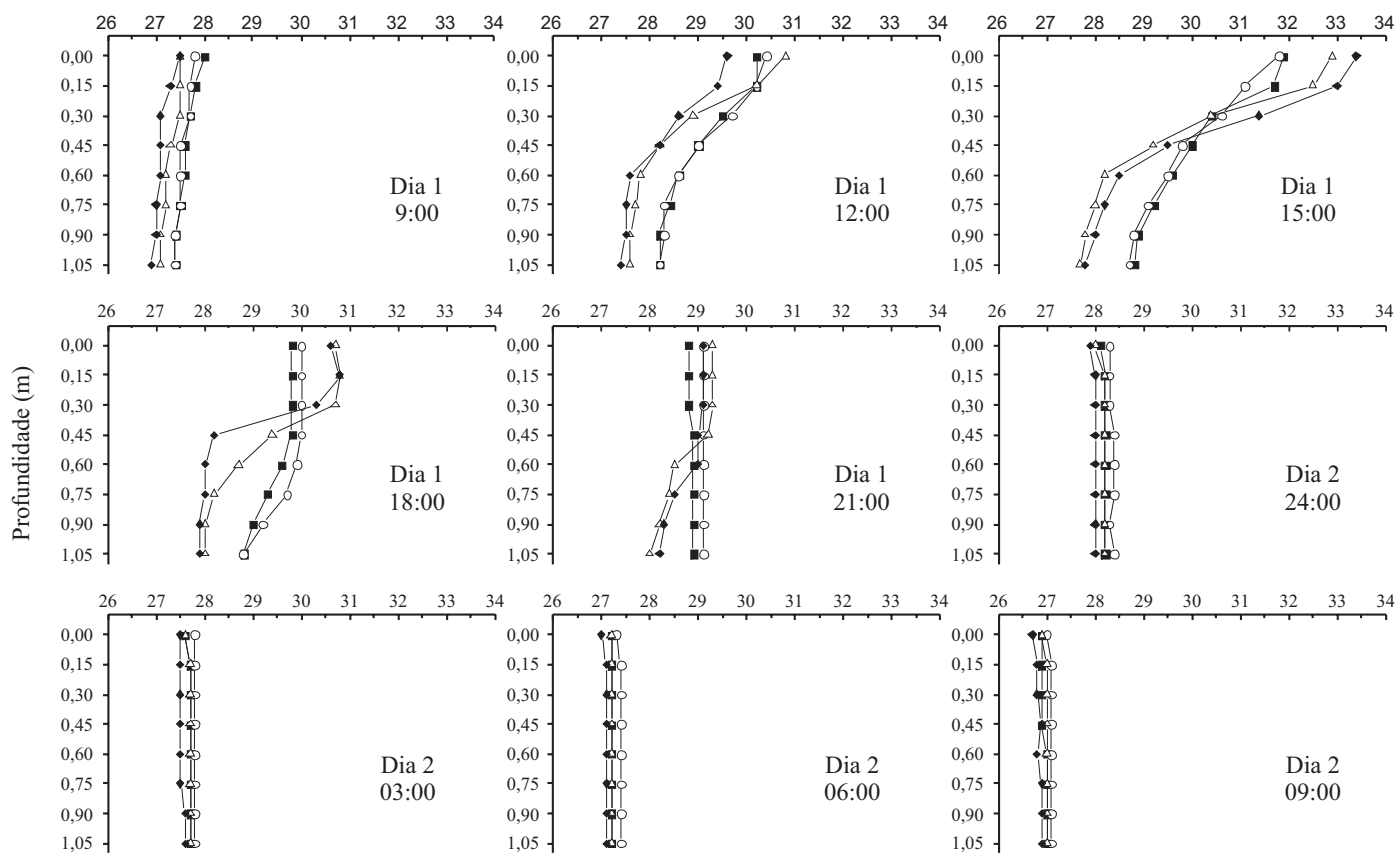

= 40 indivíduos $/ \mathrm{m}^{2} ; \bigcirc=60$ indivíduos $/ \mathrm{m}^{2} ; \Delta=80$ indivíduos $/ \mathrm{m}^{2} ;=100$ indivíduos $/ \mathrm{m}^{2}$

Figura 2 - Variação diária (padrão nictemeral) dos perfis verticais de temperatura de viveiros de camarões-da-amazônia no mês de fevereiro de 2008. 


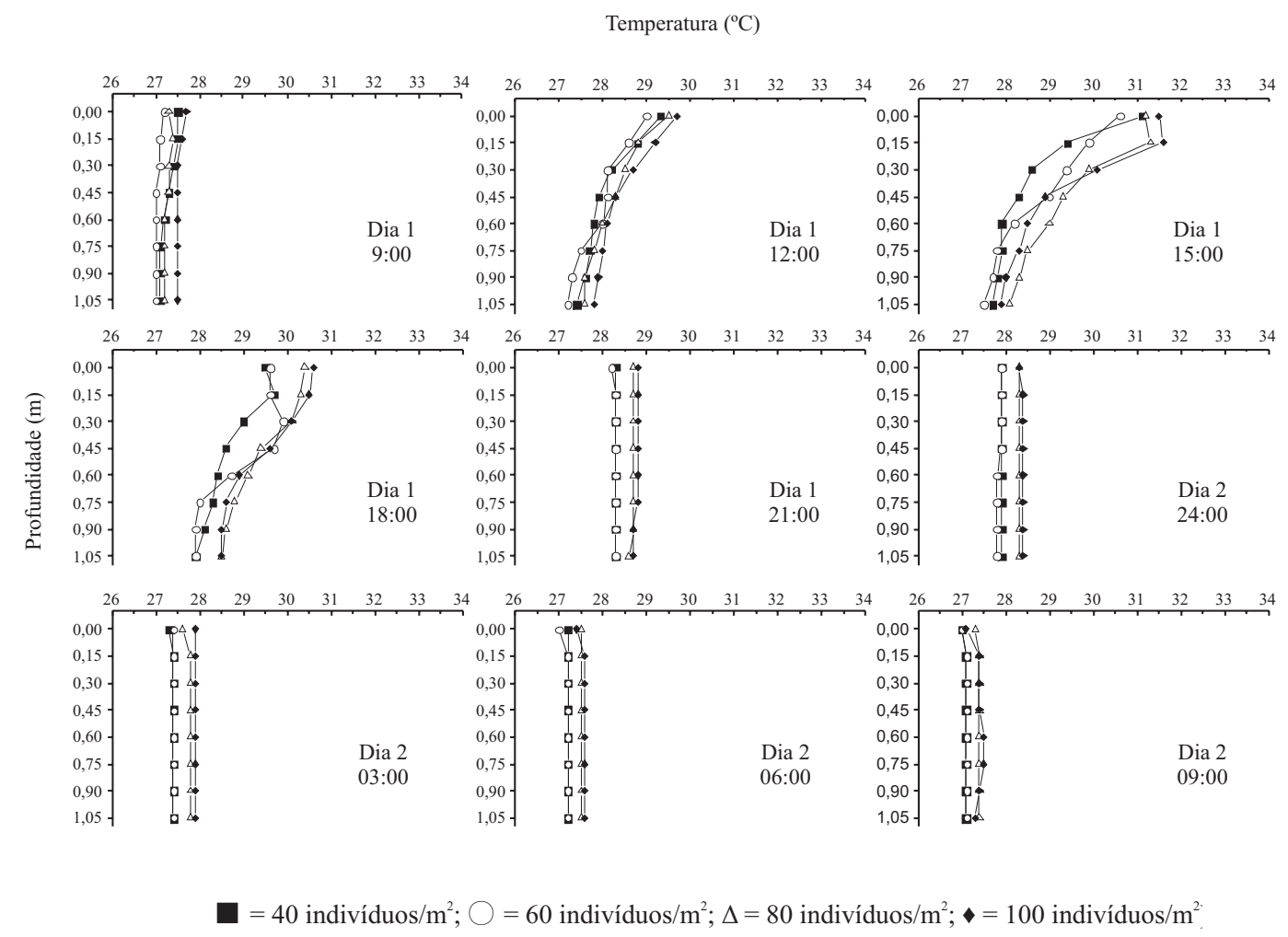

Figura 3 - Variação diária (padrão nictemeral) dos perfis verticais de temperatura dos viveiros de camarões-da-amazônia no mês de março de 2008.

Saturação de oxigênio (\%)
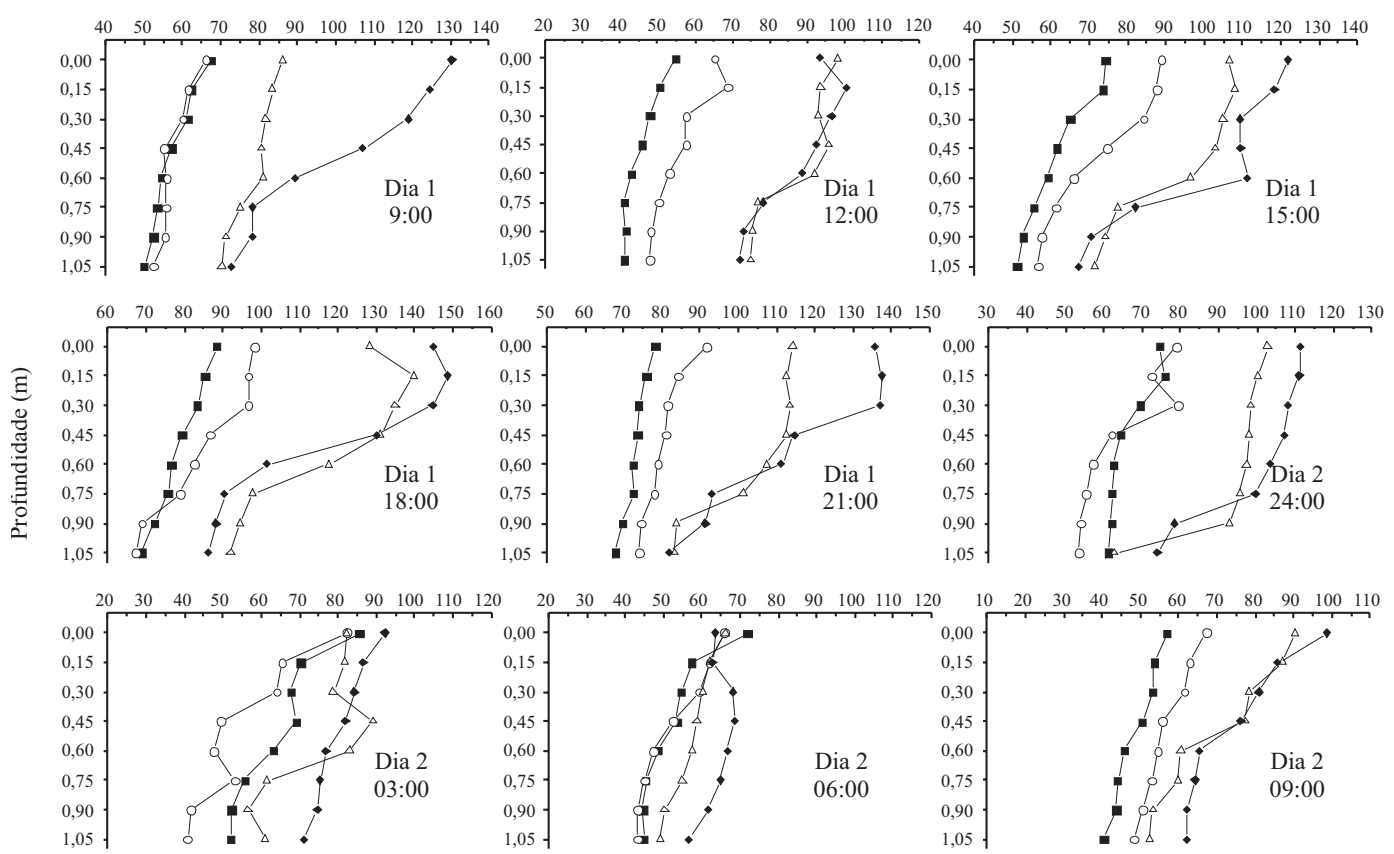

= 40 indivíduos $/ \mathrm{m}^{2} ; \bigcirc=60$ indivíduos $/ \mathrm{m}^{2} ; \Delta=80$ indivíduos $/ \mathrm{m}^{2} ; \bullet=100$ indivíduos $/ \mathrm{m}^{2}$

Figura 4 - Variação diária (padrão nictemeral) dos perfis verticais de saturação de oxigênio dissolvido de viveiros de criação de camarõesda-amazônia no mês de janeiro de 2008. 
Saturação de oxigênio (\%)

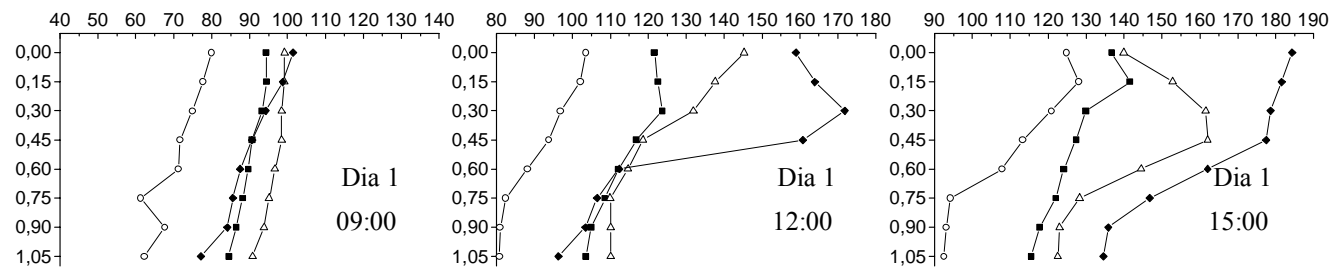

$90100110120130140150160170180190 \quad 70 \quad 8090100110120130140150160170 \quad 50 \quad 6070 \quad 8090100110120130140150$
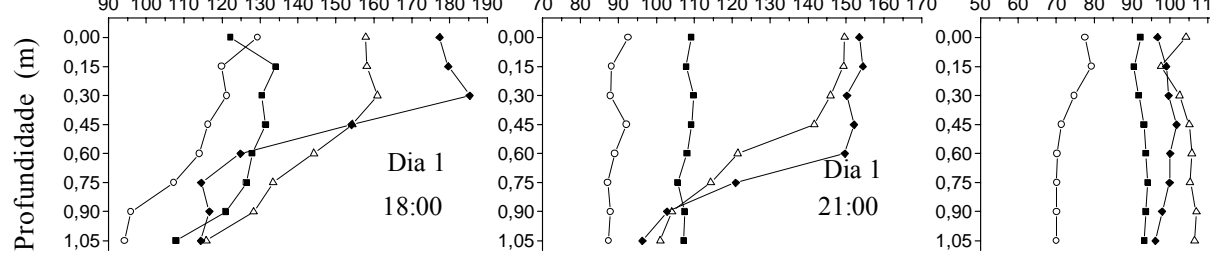

Dia 2

$24: 00$

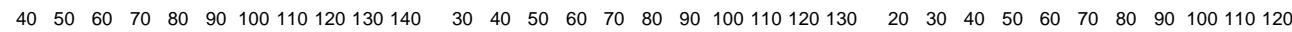

Dia 2

I = 40 indivíduos $/ \mathrm{m}^{2} ; \bigcirc=60$ indivíduos $/ \mathrm{m}^{2} ; \Delta=80$ indivíduos $/ \mathrm{m}^{2} ; \bullet=100$ indivíduos $/ \mathrm{m}^{2}$

Figura 5 - Variação diária (padrão nictemeral) dos perfis verticais de saturação de oxigênio dissolvido de viveiros de camarões-da-amazônia no mês de fevereiro de 2008.

Saturação de oxigênio (\%)
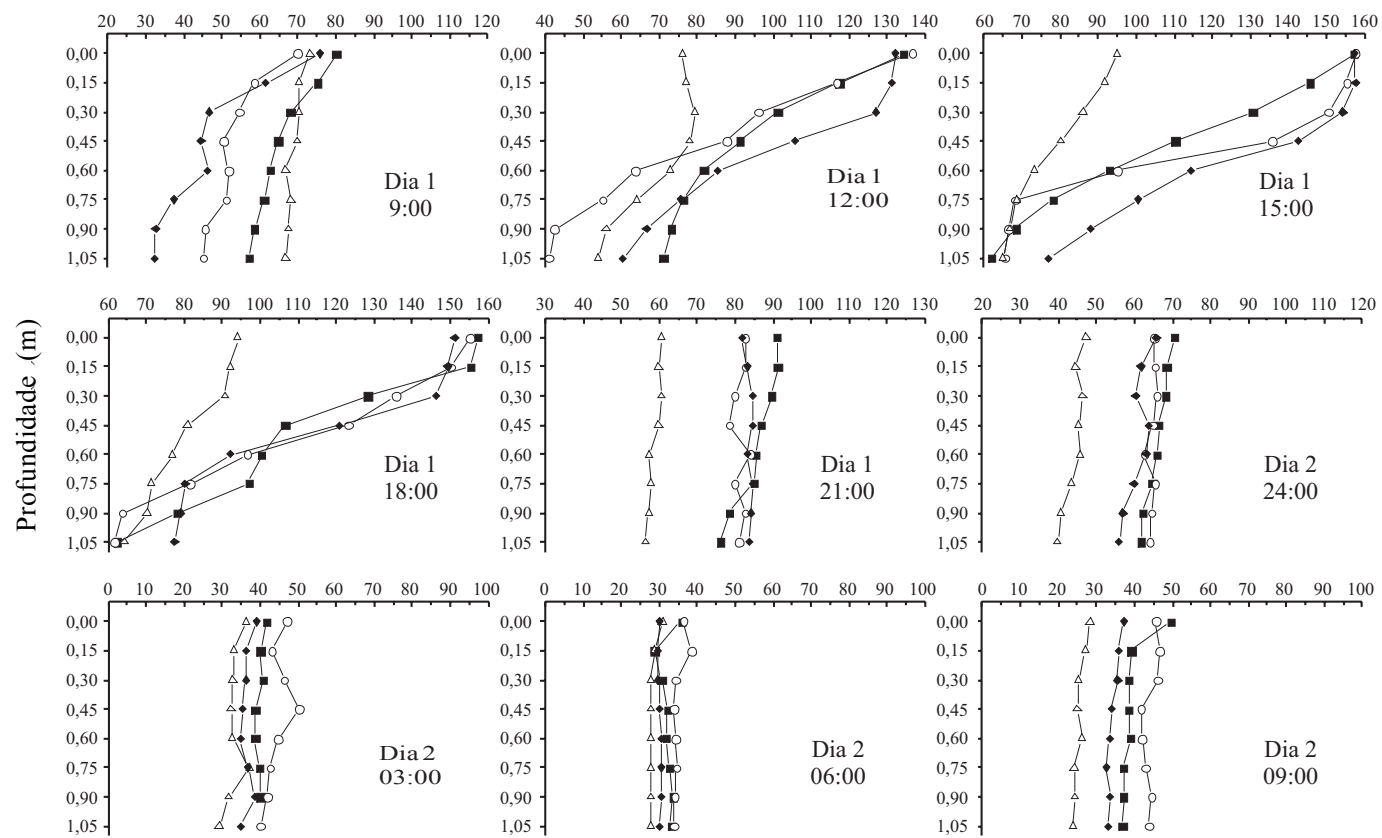

= 40 indivíduos $/ \mathrm{m}^{2} ; \bigcirc=60$ indivíduos $/ \mathrm{m}^{2} ; \Delta=80$ indivíduos $/ \mathrm{m}^{2} ; \star=100$ indivíduos $/ \mathrm{m}^{2}$

Figura 6 - Variação diária (padrão nictemeral) dos perfis verticais de saturação de oxigênio dissolvido dos viveiros de camarões-daamazônia no mês de março de 2008. 
de oxigênio pelos organismos aquáticos durante a noite. Entretanto, em nenhum momento, ocorreu a anoxia da coluna d'água em qualquer das densidades de cultivo. Os menores valores saturação de oxigênio no fundo dos viveiros em nenhum momento foram inferiores a $23 \%$.

A maior saturação de oxigênio encontrada durante todo o experimento foi em fevereiro, às $18 \mathrm{~h}$, no viveiro com maior densidade de cultivo (185\%). Esse valor foi encontrado logo abaixo da superfície e provavelmente refletiu o processo de migração do fitoplâncton para regiões da coluna d'água que não recebem incidência elevada de raios solares. De fato, Giani \& Leonardo (1988) estudaram a distribuição vertical de algas fitoplanctônicas no reservatório da Pampulha, Minas Gerais, e observaram maiores densidades desses organismos logo abaixo da superfície.

Em todas as densidade de cultivo, os viveiros apresentaram padrão de circulação do tipo polimítico, com circulações frequentes. Sá-Junior \& Sipaúba-Tavares (1997) avaliaram a variação diária de parâmetros limnológicos em tanques de cultivo planctônico e também identificaram ao longo do dia os processos de estratificação e circulação da coluna d'água, semelhante a ecossistemas aquáticos polimíticos. Neste trabalho, esse padrão de circulação provavelmente foi ocasionado pela pouca profundidade dos viveiros e pelo resfriamento da camada superficial da coluna d'água durante a noite e pelo seu intenso aquecimento durante o dia.

A análise dos componentes principais resumiu $85,57 \%$ da variabilidade total dos dados em seus dois primeiros componentes: o primeiro componente explicou $65,44 \%$ do total da variação encontrada, enquanto o segundo explicou 20,13\%. As variáveis P-total, $\mathrm{N}$-total P-ortofosfato, $\mathrm{N}$-amoniacal e turbidez foram negativamente correlacionadas ao componente principal 1 , enquanto $\mathrm{N}$-nitrato foi correlacionado positivamente. N-nitrito esteve correlacionado negativamente ao componente 2 . Todas as variáveis apresentaram correlacionadas a algum dos dois componentes, pelo valor correlação de estabelecido em 0,6 (Tabela 2; Figura 7).

A densidade de 100 indivíduos $/ \mathrm{m}^{2}$ (agrupamento $\mathrm{C}$ ) encontra-se mais à esquerda do gráfico da análise dos componentes principais, apresentando maiores valores das variáveis que estão negativamente correlacionadas com o componente principal 1 (P-total, N-total, P-orto, $\mathrm{N}$-amoniacal e turbidez) e os menores valores das variáveis que estão positivamente correlacionadas com o mesmo componente (N-nitrato). A densidade 40 (agrupamento $\mathrm{A}$ ) está mais à direita do gráfico e apresenta características opostas, ou
Tabela 2 - Coeficientes de correlação entre as variáveis analisadas e os dois primeiros componentes da ordenação na análise dos componentes principais em viveiros de camarões-da-amazônia

\begin{tabular}{lcc}
\hline & \multicolumn{2}{c}{ Componente } \\
& 1 & 2 \\
\hline P-total & $-0,914622$ & 0,293989 \\
P-ortofosfato & $-0,947406$ & 0,061176 \\
N-total & $-0,674112$ & $-0,644804$ \\
N-nitrato & 0,909350 & 0,168914 \\
N-nitrito & 0,399594 & $-0,861673$ \\
N-amoniacal & $-0,770215$ & $-0,272423$ \\
Turbidez & $-0,922393$ & 0,137609 \\
\hline \% de variação explicada & 65,44 & 20,13 \\
\hline
\end{tabular}

seja, possui valores menores de P-total, N-total, P-orto, $\mathrm{N}$-amoniacal e turbidez e maiores de $\mathrm{N}$-nitrato. As densidades 60 e 80 (agrupamento B) estão no centro do gráfico, apresentando valores intermediários das variáveis analisadas.

O aumento das concentrações de nutrientes, à exceção de nitrato, no efluente dos viveiros com maior densidade de cultivo (Tabela 3 ) provavelmente esteve associado ao aumento da ração fornecida, à decomposição de matéria orgânica acumulada e à excreção dos camarões. Baccarin \& Camargo (2005) avaliaram o impacto ambiental da produção de tilápias-do-nilo em diferentes manejos alimentares e verificaram que com o aumento da biomassa dos peixes ocasionou redução gradativa dos teores de oxigênio dissolvido e aumento dos valores de turbidez e de material em suspensão do efluente, independentemente da dieta fornecida. Henry-Silva \& Camargo (2006) e Henry-Silva \& Camargo (2008) também observaram que o aumento do fornecimento de ração, associado ao crescimento dos organismos aquáticos, aumentou as concentrações de nutrientes e reduziu os valores de oxigênio dissolvido nos efluentes. Em ambientes aquáticos da Tailândia, foram constatados problemas relacionados à grande quantidade de nutrientes e de matéria orgânica produzidos pelas atividades de carcinicultura (Chua et al., 1989). Figueiredo et al. (2005) estudaram os impactos ambientais das atividades de aqüicultura e também observaram elevação na turbidez e nos níveis de fósforo total e amônia total nos efluentes de carcinicultura, principalmente quando passavam por processos de fertilização. Os resultados obtidos no presente trabalho confirmam os da literatura, pois evidenciam os efeitos da aquicultura no enriquecimento de nutrientes de seus efluentes, em decorrência das atividades de criação. 


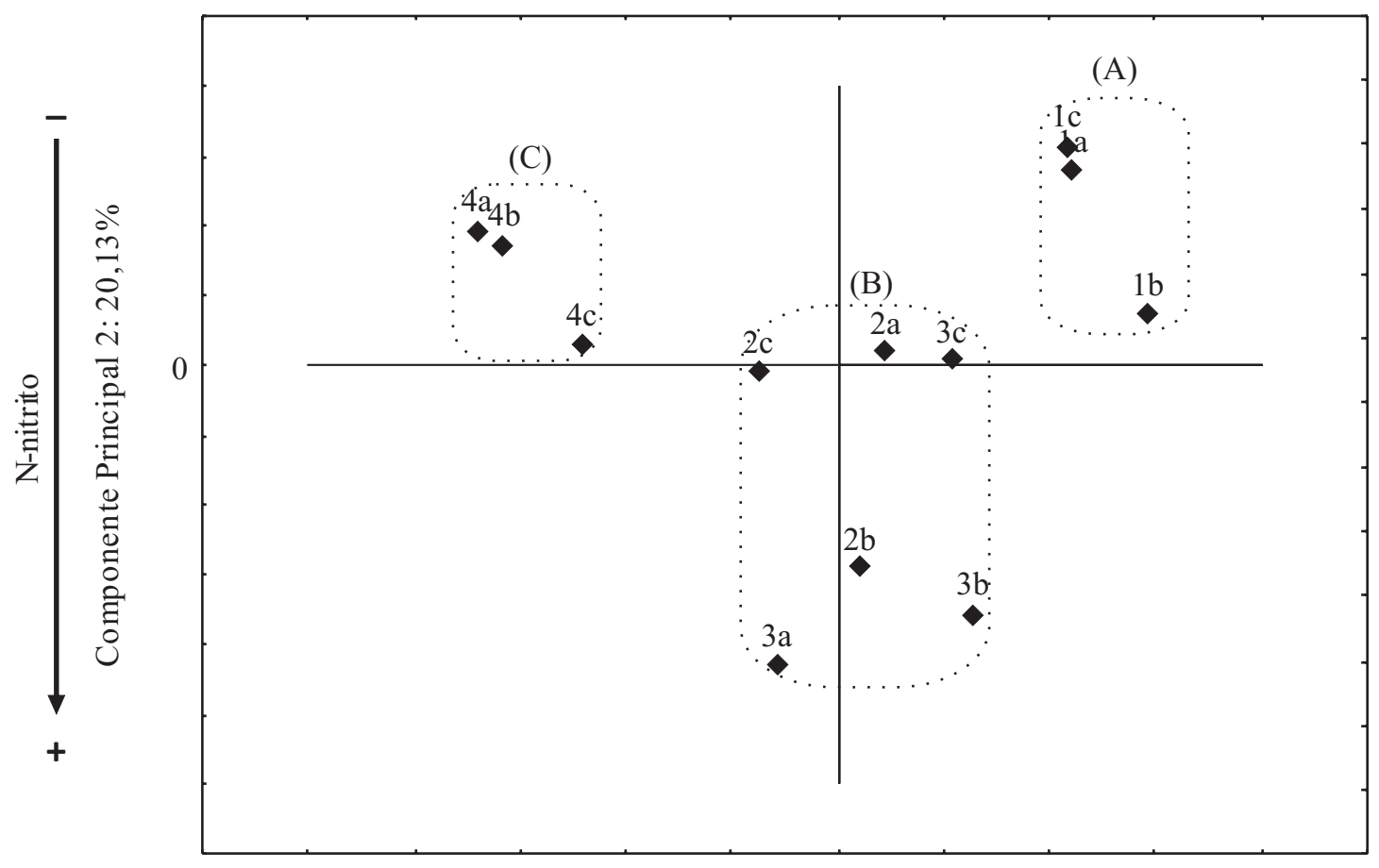

0

Componente Principal 1: 65,44\%

P-tot al, N-total, P-orto, N-amoniacal e Turbidez

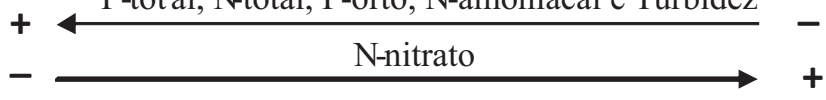

A, B e C $=$ formação de três agrupamentos; $1,2,3$ e $4=$ densidades de $40,60,80$ e 100 indivíduos $/ \mathrm{m}^{2} ;$ a, b e c $=$ réplicas de cada densidade de cultivo. Os dois componentes explicam $85,3 \%$ da variação observada (componente principal $1=65,44 \%$; e componente principal $2=20,13 \%$ )

Figura 7 - Ordenação pela análise dos componentes principais das variáveis limnológicas dos efluentes de camarões-da-amazônia criados em quatro densidades.

Tabela 3 - Valores médios (três meses) de nitrogênio e fósforo e turbidez na água de abastecimento e no efluente de viveiros com diferentes densidades de cultivo de camarões-da-amazônia. Densidade 40 (40 indivíduos $\left./ \mathrm{m}^{2}\right)$, Densidade $60\left(60\right.$ indivíduos $\left./ \mathrm{m}^{2}\right)$, Densidade $80\left(80\right.$ indivíduos $\left./ \mathrm{m}^{2}\right)$ e Densidade $100\left(100\right.$ indivíduos $\left./ \mathrm{m}^{2}\right)$

\begin{tabular}{lcrrrr}
\hline & & \multicolumn{3}{c}{ Densidade de cultivo } \\
\hline Variável & Abastecimento & 40 indivíduos $/ \mathrm{m}^{2}$ & 60 indivíduos $/ \mathrm{m}^{2}$ & \multicolumn{1}{c}{80 indivíduos $/ \mathrm{m}^{2}$} & 100 indivíduos $/ \mathrm{m}^{2}$ \\
\hline N-total $\left(\mathrm{mg} . \mathrm{L}^{-1}\right)$ & $0,22 \pm 0,01$ & $0,28 \pm 0,02$ & $0,32 \pm 0,00$ & $0,36 \pm 0,02$ & $0,38 \pm 0,01$ \\
N-amoniacal $\left(\mu \mathrm{g} . \mathrm{L}^{-1}\right)$ & $12,72 \pm 1,10$ & $13,55 \pm 0,46$ & $14,44 \pm 1,87$ & $17,75 \pm 6,01$ & $19,79 \pm 1,31$ \\
N-nitrato $\left(\mu \mathrm{g} . \mathrm{L}^{-1}\right)$ & $177,58 \pm 12,02$ & $163,03 \pm 1,48$ & $147,36 \pm 5,97$ & $125,07 \pm 11,73$ & $88,70 \pm 5,12$ \\
N-nitrito $\left(\mu \mathrm{g} . \mathrm{L}^{-1}\right)$ & $19,52 \pm 1,30$ & $22,40 \pm 2,34$ & $20,84 \pm 3,93$ & $21,27 \pm 1,98$ & $16,61 \pm 2,41$ \\
P-total $\left(\mu \mathrm{g} . \mathrm{L}^{-1}\right)$ & $86,40 \pm 2,58$ & $138,77 \pm 2,30$ & $128,32 \pm 1,58$ & $167,95 \pm 1,42$ & $220,21 \pm 3,27$ \\
P-ortofosfato $\left(\mu \mathrm{g} . \mathrm{L}^{-1}\right)$ & $4,69 \pm 0,31$ & $6,00 \pm 0,74$ & $19,53 \pm 1,69$ & $15,15 \pm 0,75$ & $58,49 \pm 1,66$ \\
Turbidez $(\mathrm{NTU})$ & $33,42 \pm 2,04$ & $37,42 \pm 1,01$ & $45,25 \pm 16,49$ & $49,08 \pm 0,80$ & $56,08 \pm 18,38$ \\
\hline
\end{tabular}




\section{Conclusões}

$\mathrm{Na}$ coluna d'água de viveiros de camarões-daamazônia, independentemente da densidade de cultivo, ocorrem estratificações e desestratificações diárias da temperatura e da saturação de oxigênio, que indicam padrão de circulação polimítico dessa espécie. A coluna d'água dos viveiros de Macrobachium amazonicum, independentemente da densidade de cultivo, apresenta perfil de oxigênio do tipo clinogrado. Efluentes de viveiros com maiores densidades de cultivo apresentam valores mais elevados de $\mathrm{P}$-total, $\mathrm{N}$-total, $\mathrm{P}$-orto, $\mathrm{N}$-amoniacal e turbidez.

\section{Agradecimentos}

Ao Prof. Dr. Wagner Cotroni Valenti e à Dra. Patrícia M. C. Moraes-Valenti, responsáveis pelo Setor de Carcinicultura do Centro de Aquicultura da UNESPJaboticabal, pelo auxílio nas atividades experimentais, pelas valiosas sugestões e por gentilmente terem cedido as instalações do setor. À CAPES, pela bolsa de estudo oferecida por intermédio do programa PRODOC ao primeiro autor.

\section{Referências}

BACCARIN, A.E.; CAMARGO, A.F.M. Characterization and evaluation of the impact of feed management on the effluents of Nile tilapia (Oreochromis niloticus) culture. Brazilian Archives of Biology and Technology, v.48, p.81-90, 2005.

BOYD, C. Guidelines for aquaculture effluent management at the farm-level. Aquaculture, v.226, p.101-112, 2003.

BOYD, C.E.; ZIMMERMANN, S. Grow-out systems: water quality and soil management. In: NEW, M.B.; VALENTI, W.C (Eds.). Freshwater prawn culture: The farming of Macrobrachium resenbergii. Oxford: Blackwell Science, 2000. p.221-434.

CHUA, T.E.; PAW, J.N.; GUARIAN, F.Y. The environmental impact of aquaculture and the effects of pollution on coastal aquaculture development in southeast Asia. Marine Pollution Bulletin, v.20, p.335-343, 1989.

ESTEVES, F.A.; BOZZELLI, R.L.; CAMARGO, A.F.M. et al. Variação diária (24 horas) de temperatura, oxigênio dissolvido, $\mathrm{pH}$ e alcalinidade em duas lagoas costeiras do estado do Rio de Janeiro e suas implicações no metabolismo destes ecossistemas. Acta Limnológica Brasiliensia, v.2, p.99-127, 1988.

FIGUEIREDO, M.C.B.; ARAUJO, L.F.P.; GOMES, R.B. et al. Impactos ambientais do lançamento de efluentes da carcinicultura em águas interiores. Engenharia Sanitária Ambientental, v.10, n.2, p.167-74, 2005.

GIANI, A.; LEONARDO, I.M. Distribuição vertical de algas fitoplânctonicas no reservatório da Pampulha (Belo Horizonte, MG). Acta Limnologica Brasiliensia, v.2, p.387-404, 1988.

GOLTERMAN, H.L.; CLYNO, R.S.; OHNSTAD, M.A.M. Methods for physical and chemical analysis of fresh water. London: Blackwell Science Publication, 1978. 214p. (IBP Handbook, 8).

HENRY-SILVA, G.G.; CAMARGO, A.F.M. Efficiency of aquatic macrophytes to treat Nile tilapia pond effluents. Scientia Agrícola, v.63, p.433-438, 2006.

HENRY-SILVA, G.G.; CAMARGO, A.F.M. Tratamento de efluentes por macrófitas aquáticas flutuantes. Revista Brasileira de Zootecnia, v.37, n.2, p.181-188, 2008.

KOROLEFF, F. Determination of nutrients. In: GRASSHOFF, K. (Ed.) Methods of seawater analysis. Wwinhein: Verlog Chemic, 1976. p.117-181.

KUBITZA, F. Qualidade da água no cultivo de peixes e camarões. 3.ed. Jundiaí: ESALQ/USP, 2003, 229p.

LUDWIG, J.A.; REYNOLDS, J.F. Statistical ecology: a primer on method and computing. London: John Wiley, 1988. 337p.

MACKERETH, F.I.F.; HERON, J.; TALLING, J.F. Water analysis: some revised methods for limnologist. London: Freshwater Biological Association, 1978. 121p.

MIDLEN, A.; REDDING, T. Environmental management for aquaculture. Netherlands: Kluwer Academic Publishers, 1998. 223p.

MIRES, D. Aquaculture and the aquatic environment: mutual impact and preventive management. The Israeli Journal of Aquaculture, v.47, p.163-172, 1995.

MORAES-RIODADE, P.M.C.; VALENTI, W.C. Morphotypes in male Amazon river prawns, Macrobrachium amazonicum. Aquaculture, v.236, n.1-4, p.297-307, 2004.

MORAES-VALENTI, P.M.C.; VALENTI, W.C. Effect of intensification on grow out of the Amazon river prawn, Macrobrachium amazonicum. Journal of the World Aquaculture Society, v.28, n.4, p.516-526, 2007.

NACA/FAO. Aquaculture development beyond 2000: the Bangkok declaration and strategy. In: CONFERENCE ON AQUACULTURE IN THE THIRD MILlENNIUM, Bangkok. Proceedings... Bangkok: NACA, Bangkok \& FAO, 2000. 27p.

NEW, M.B. Freshwater prawn farming: global status, recent research and a glance at the future. Aquaculture Research, v.36, p.210-230, 2005.

PAYNE, A.I. The ecology of tropical lakes and rivers. Chichester: John Wiley \& Sons, 1986. 301p.

RIMON, A.; SHILO, M. Factors which affect the intensification of fish breeding in Israel. I. Phisical, chemical and biological characteristics of the intensive fish ponds in Israel. Bamidgeh, v. 34, n. 3, p. $87-100,1982$

SÁ-JUNIOR, W.P.; SIPAÚBA-TAVARES, L.H. Produtividade primária fitoplanctônica e variação de parâmetros limnológicos ao longo do dia, em tanques de cultivo planctônico da estação de hidrobiologia e piscicultura de Furnas. Acta Limnológica Brasiliensia, v.9, p.83-91, 1997.

VALENTI, W.C.; MORAES-RIODADES, P.M.C. Freshwater prawn farming in Brazil. Global Aquacultures Advocate, v.7, n.4, p. 52-53, 2004.

VALENTI, W.C. Introdução. In: VALENTI, W.C.; POLI, C.R.; PEREIRA, J.A. et al. (Eds.). Aqüicultura no Brasil: bases para um desenvolvimento sustentável. Brasília: CNPQ/MCT, 2000. p.25-32. 